\title{
A Comparative Simulation of Opportunistic Routing Protocols Using Realistic Mobility Data Obtained from Mass Events
}

\author{
Arno Barzan*, Bram Bonné*, Peter Quax*, Wim Lamotte* \\ ${ }^{*}$ Hasselt University - tUL - iMinds \\ Wetenschapspark 2 \\ 3590 Diepenbeek, Belgium \\ Email: arno.barzan@uhasselt.be
}

\author{
Mathias Versichele ${ }^{\dagger}$, Nico Van de Weghe ${ }^{\dagger}$ \\ ${ }^{\dagger}$ Department of Geography, Ghent University \\ Krijgslaan 281 (S8) \\ 9000 Ghent, Belgium
}

\begin{abstract}
Large crowds at music festivals or other mass events create challenging environments for traditional infrastructure based wireless networks. Mobile devices carried by the attendees produce large amounts of network traffic that can result in network outage or serious delays. Opportunistic networks may offer solutions to enable communication between attendees and/or organizers through direct communication between devices, without requiring a fixed infrastructure. In previous work, researchers have developed numerous opportunistic routing protocols designed to enable communication in such ad hoc networks. In this paper, several of these protocols are evaluated for the specific use case of a music festival by running simulations that make use of realistic mobility data collected during an international music festival. This analysis allows for identification of candidate routing protocols that exhibit properties that make them suitable for the envisaged scenario. The goal is to narrow down the set of candidate protocols and to eventually fine tune them to optimize their working. Based on the simulations, we propose two candidate routing protocols that are most suited for use at mass events.
\end{abstract}

Keywords-ad hoc networks; simulation; routing protocols; mobile communication

\section{INTRODUCTION}

The existing cellular network infrastructure at mass events is often faced with very large amounts of data, which can result in network outage or serious delays, especially in places where many people gather in a small geographical area. The main goal of this research is to enable attendees and/or organisers to exchange small messages (e.g. SMS or WhatsApp) through ad hoc communication at mass events like music festivals, reducing the load and freeing capacity on traditional cellular networks. Opportunistic network technology is leveraged to directly deliver data between devices.

Crowds at mass events follow specific movement patterns, the properties of which can be exploited to better route information between users. In literature, several routing protocols have been proposed which can be used to implement such an ad hoc messaging application on mobile devices. All of these protocols require different parameters to be tuned. These choices range from how long one should wait for the arrival of a message to the number of messages that should be passed on to a neighboring device. Simulation is a common tool in research on computer networks, especially in Mobile Ad
Hoc Network (MANET) and Delay Tolerant Network (DTN) research. In this paper, we present the results of simulations of these various routing protocols using the Opportunistic Network Environment (ONE) Simulator [1] with mobility data which was captured at an international music festival. Furthermore, by analysing the data, a suggestion for best-suited protocols will be made for an actual implementation.

\section{BACKGROUND AND RELATED WORK}

In the last decade of DTN research the main focus has been on the development of efficient routing protocols. Several surveys can be found in literature [2], [3]. A commonly used tool to evaluate these protocols is simulation. There are several available network simulators, for example ns-2 (and its successor ns-3) [4] or OMNeT++ [5], each with its own benefits and drawbacks. The Opportunistic Networking Environment (ONE) simulator presented by Keränen and Ott [1], is specifically designed to facilitate DTN related simulations and is used and evaluated in several publications.

Although simulations are a commonplace tool in research, there are typical mistakes and misconceptions. Andel and Yasinsac [6] point out several of these in the reporting of simulation studies. More specifically, Grasic and Lindgren [7] have surveyed a number of DTN related papers published in the last decade and describe several issues that should be avoided in future research, with the emphasis on using the correct node density and mobility models for a given scenario.

Aschenbruck et al. [8] provide an overview of the available synthetic and trace-based mobility models that can be used in simulations and emphasize the need for more realistic traces and mobility models. The CRAWDAD database [9] is a well known repository in the domain and contains diverse wireless trace data sets which can be used to extract mobility information. Numerous publications using those traces to improve synthetic models can be found, for example Lee et al. [10] who developed a movement model based on GPS traces. Other publications use the aforementioned traces to evaluate a protocol, like the work of Radenkovic and Grundy [11] wherein several traces are used to evaluate a proposed routing strategy.

We have used the method of Versichele et al. [12] to gather a large set of mobility traces at an international music festival, 
and have used this data to simulate and investigate existing DTN protocols in a mass event setting without the need to depend on synthetic models. Vukadinovic and Mangold [13] performed an analogous experiment in an entertainment park where GPS traces were collected using mobile devices that were randomly distributed to visitors over five days. However, they did not focus on different routing protocols using these traces.

\section{MoBILITY}

The chosen mobility model has a profound impact on the realism and thus outcome of a simulation. As Grasic and Lindgren [7] have pointed out, many protocols in the literature are tested and evaluated in unrealistic or irrelevant scenarios. This paper focuses on a specific real-world scenario and the development of applications for it.

\section{A. Collecting Data}

Despite projects like CRAWDAD, the public availability of wireless traces is rather limited. For this study, a subsample of festival attendees was tracked using the method of Versichele et al. Using this method, the complex movement patterns of attendees at a mass event can be captured. By deploying 15 Bluetooth scanners at strategic locations over the festival terrain, proximity-based trajectories of over 10000 unique devices were registered during the Pukkelpop 2012 festival. This is a three-day Belgian music festival attracting 100000 visitors each year and featuring many international acts. The unique hardware addresses (MAC) of Bluetooth chips make it possible to detect and track the movement of a device when combining data from several detectors. The hardware address can also be used to couple a detected device to a node that is used in a simulation environment. The resulting data contains timestamps of detected devices collected at certain strategically chosen locations on the terrain, such as stages and passageways, while trying to cover as much of the terrain as possible.

\section{B. Processing}

Paths can be deduced from the above data by letting a node travel from a certain detection location and time to the location and time of the next detection. The range of the detectors, depending on the sensor used, is approximately 20 30 meters. Nodes in the simulation are therefore placed at or move towards a random position around the sensor locations in a radius of 20 meters.

If the nodes would simply travel in straight lines from one detection to another, certain obstacles that were present at the festival area would be ignored. To improve realism, a rudimentary shortest path finding algorithm was used to let nodes travel a more realistic path from one point to the next: paths are only created along passageways, since fences and tents on the terrain can not be crossed. To achieve this, additional points were added along paths in order to avoid these obstacles.

In between detections it is possible that certain devices remain undetected for a while. This does not necessarily mean these users left the festival area or turned off their devices: they could simply be out of range of all detectors. The problem is

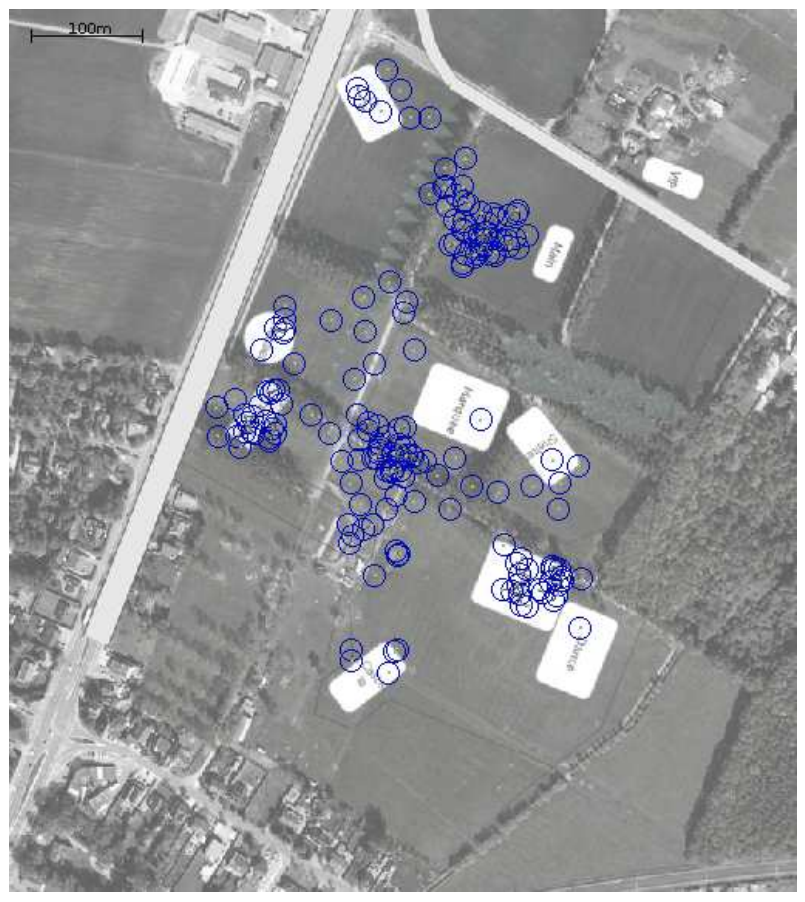

Fig. 1. Simulation of a festival day: the nodes and their transmission ranges are visualized by a circle, the background shows the festival area.

that there is no other information about the actual status of the device. The chosen solution to this problem is to disable the node in the simulator after a certain time of not being detected. This time was empirically chosen to be 15 minutes: most of the festival area is covered and it should take less than 15 minutes to move between any two detectors. The node will remain disabled until a detection at a later timestamp is encountered in the data.

The Bluetooth detectors discovered an average of 5300 unique devices per festival day. This number also includes devices that are only detected a few times (e.g. a passer-by) or are originating from organisational equipment which is static. A set of 1000 nodes with the best paths (i.e. paths with the most detection points) were selected for the simulations. We have performed simulations with 50, 100, 250, 500 and 1000 nodes.

\section{Simulations}

The main goal of all evaluated protocols is to enable communication between arbitrary devices in an ad hoc manner. This means that messages must be relayed by other devices to reach destinations that are not in transmission range. This section briefly explains the settings that were used for the simulations, followed by a description of the evaluated protocols.

\section{A. Simulation Settings}

The ONE simulator is a network simulator developed to evaluate network protocols in a delay-tolerant environment. It is specifically designed to facilitate DTN research by abstracting certain elements of typical DTN communication and offering a number of tools for simulation. Visualization is one of these tools; Figure 1 shows a screenshot of the visualization 
component while simulating moving nodes on the festival area. Another tool enables researchers to import mobility data into the simulator. The source code of this component had to be slightly altered so nodes could be disabled when no trace data was available (as explained in section III-B). Messages in the buffers of these disabled nodes are kept there but do age; depending on the routing protocol, it is possible they are dropped from the buffer due to aging as would be the case when devices have left the festival site in a real-world scenario. Each simulation run covers a full festival day (12 hours).

The size of the festival area is circa $400 \mathrm{~m}$ by $500 \mathrm{~m}$. For all nodes in the simulations a communication range of ten meters was chosen using a Bluetooth interface with a transfer rate of $2 \mathrm{Mbit} / \mathrm{s}$. Although many wireless technologies have a much larger range in theory, interference in a dense crowd and other obstacles often hinder communication over a large distance. The ONE simulator does not take into account these interferences; a relatively small transmission range is chosen to compensate for this deficiency, so the results are also relevant for other wireless technologies (e.g. WiFi) that can be used in a real-world application.

The messages in the simulations have a size of $100 \mathrm{kB}$ and the buffers in each node have a capacity of $10 \mathrm{MB}$. This is an estimation of the requirements of an application on a mobile device for sending small text messages or pictures like in SMS/MMS. The size of the messages is large enough to support additional security measures such as the use of public-key cryptography. The buffer size is intentionally kept small (a maximum of 100 messages): more messages lead to more processing and transmissions and thus more energy consumption. People who do not return home between festival days have to consider their energy consumption since they often have no way of recharging their devices. This factor should be taken into account when developing and testing routing protocols for scenarios like a festival spanning several days.

Messages are generated by random active nodes in the festival area. A node is assumed to be active when the simulator is certain of the node's location, i.e. when there is a related detection in the real-world trace for that particular node. The destination is also a random node in the festival area. Because nodes can become disabled, it is possible that the associated messages will disappear as well. This is not unrealistic since people may turn off their devices, the application is shut down or because batteries could be depleted in real life. Using a random event generator in our simulations, every node sends an average of four messages per hour in a simulated festival day.

The time-to-live (TTL) of the messages was set to 15 minutes. This is rather a long time to mimic SMS-like behavior but it ensures that all protocols have a reasonable amount of time to deliver a message. In an actual application this TTL should be further reduced since an acknowledgement system should inform the user whether a sent message has arrived at its destination, and long waiting times for this feedback are impractical.

\section{B. Routing Protocols}

Six protocols were tested in the simulations. Every protocol was run through five iterations of simulation, each iteration simulating a full festival day of 12 hours, with the results being averaged. In each of the five runs a different seed was used for the random number generator responsible for the time of creation, sender and destination of the messages. The protocols used were Direct Delivery (DD), First Contact (FC), Epidemic (EP) [14], Spray and Wait (SW and SWb) [15] and two versions of the Probabilistic Routing Protocol (PRoPHET): the original protocol (PR) [16] and a slightly adjusted version PRoPHETv2 (PR2) [17]. The implementations of these protocols were made by the developers of the ONE simulator and other contributors. The active community and diverse research contributions have checked and optimized these implementations. Since the goal was to investigate general properties of routing algorithms in a specific environment, the choice was made to use specifically these thoroughly tested and reviewed protocols. A short description of each is provided below.

The first three protocols do not have parameters that can be tuned, so there is only one version to be tested. $D D$ delivers the message only directly to the destination, i.e. there is no relaying, and messages are only delivered when the destination is a direct neighbor of the sender. In $F C$ only one copy of a message exists at a certain point in time: a message is relayed to the first encountered node unless the message already traversed that node, and is then removed from the message buffer of the previous hop. This happens until the destination is reached or the TTL is reached. The EP protocol replicates messages using a flooding approach so that all messages are constantly being sent to all nodes that are in range.

In $S W$, a maximum of $n$ copies of a message can exist in the network. These copies are 'sprayed' towards encountered nodes, until only one copy remains at the sender. Two implementations of $S W$ were used: binary $(\mathrm{SWb})$ and nonbinary (SW). In non-binary mode the sender sprays only one copy of the message to each (different) encountered node. In binary mode half of the available copies is passed on to an encountered node, which in turn does the same with these received copies. This happens until only one copy is left. The remaining copy then waits, in both binary and non-binary, to be delivered to the destination as soon as it comes into range. Both the binary and non-binary versions were simulated with different numbers of copies ( $n=6,12,18,36,72,144)$, based on values proposed in the original work and further empirically determined by preliminary simulations.

The PRoPHET protocols try to estimate the chance that a node can deliver the message to another node, based on historical information, and spread messages based on this chance. The transitivity property describes the probability by which a node can deliver a copy to the destination recursively. For example, if node A wants to send a message to node D via $B$, the transitivity property specifies the probability that $B$ will encounter a node $C$ which can in turn deliver the copy to D. $P R 2$ uses a slightly adjusted transitivity property and aging of the delivery probabilities. More details can be found in the respective papers [16], [17]. PR and PR2 ran with different values for the transitivity property $(\beta=0.0,0.09,0.25,0.90)$ based on the proposed values in original and on previous work. 


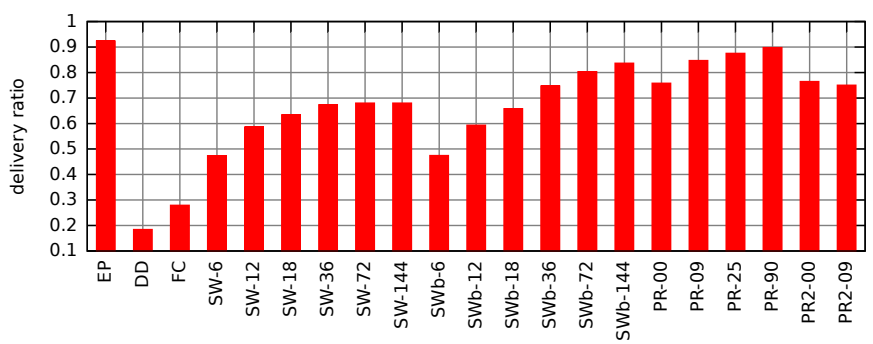

(a) Delivery ratio

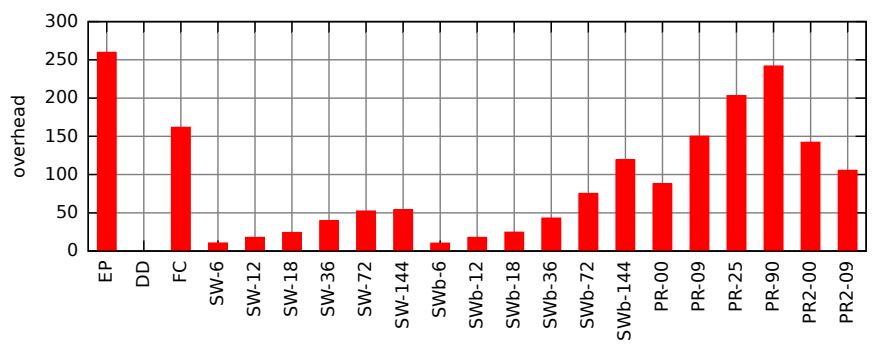

(c) Overhead

Fig. 2. Comparison between routing protocols based on the indicated metric.

\section{RESUlTS AND ANALYSIS}

This section discusses the results and analysis of the simulations described in Section IV. First, the proposed metrics are briefly described. The importance of these metrics is dependent on the application scenario and will be detailed in the analysis that follows. Finally, the most suitable protocols are suggested based on the simulations. The figures in this section show the results of the simulation runs with 500 nodes.

\section{A. Metrics}

1) Delivery ratio: This metric represents the percentage of messages that were created and effectively reached their destination.

2) Latency: The time it takes for a delivered message to reach its destination. An upper bound on the time a message has to arrive at the destination may be needed in an application to decide whether the message is lost or a resend may be beneficial.

3) Overhead: The overhead is defined as $\frac{\# \text { relayed-\#delivered }}{\# \text { delivered }}$. It provides an indication of the overhead as transmissions of messages that could not reach their destination are also included.

4) Number of hops: This number describes how many other nodes the message had to pass through to reach its final destination. This metric is often omitted or forgotten in the analysis or reporting of simulations but can indicate how challenging the evaluating scenario was and how well the routing protocol utilizes the network resources [7].

\section{B. Individual Protocol Examination}

In all of the above protocols, and more in general in the simulator used, control messages are not included in the overhead calculation. The nodes can retrieve certain information, like the history of encountered nodes in the PRoPHET algorithms, without sending actual messages. It is possible to

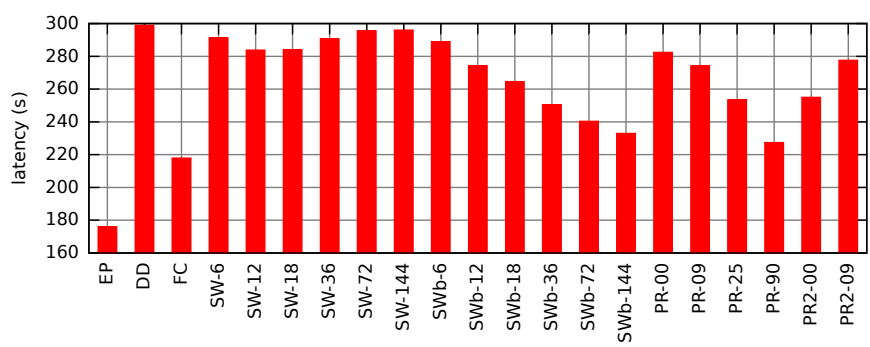

(b) Latency

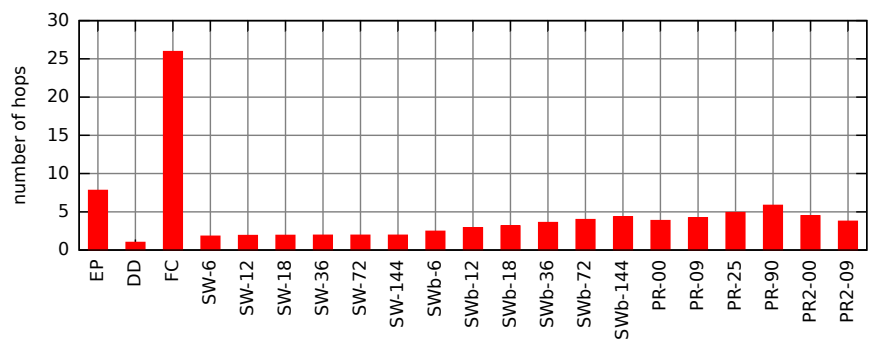

(d) Number of hops

abstract this kind of communication as is the case in the simulator, for instance by assuming the lower layers handle this, but it should not be ignored in an analysis as these messages require additional energy for processing and transmissions and can even cause additional interference.

The results of the simulations are summarized in Figure 2. What immediately stands out is the high delivery ratio of the Epidemic routing protocol. However, because of the flooding nature of this protocol, there is a relatively large overhead. It is intuitively clear that when more copies of the same message are sent, the chance of actually reaching the destination increases. The low latency of EP in this figure shows that the underlying mobility trace with 500 nodes (and similarly the 1000 trace) offers a beneficial environment for this protocol as there is a lot of movement between the groups of people at the festival site and the crowd is almost entirely interconnected.

Another part of the charts that warrants attention is the relatively large amount of hops of the First Contact protocol. The high hop count is caused by the choice of a random neighbor to relay the message, even if the destination is itself a neighbor of the sender. As can be seen on the latency graph, FC also has a relatively low end-to-end latency. The reason for this is that there is only a slight chance that the destination is reached, but if it is reached the coincidentally chosen path will be relatively fast.

The Direct Delivery protocol is not applicable for use within a real-world ad hoc application, since the point is to communicate with each other without being geographically close to each other. It is however useful to check a borderline case of DTN protocols, namely where there is no relaying at all. It is obvious that carrying messages for other nodes is necessary: the delivery property of DD is the lowest of all results. This also means that the probability is relatively small that two random nodes come in contact within 15 minutes (the chosen TTL) in the used mobility trace. 
The Spray and Wait protocols try to reduce the amount of data in the network by imposing a maximum number of copies of a certain message. It is again clear that when more copies are allowed, the delivery ratio increases. Since the non-binary version spreads a message by giving one copy to each encountered node until only a single copy remains at the sender, the number of hops is always one (if the sender delivers the message) or two (if the contact delivers it). In the binary version, the maximum number of hops increases with the number of allowed copies because encountered nodes may receive more than one copy, and they can subsequently spread all of these copies. As can be seen in the graphs, the delivery ratio is relatively high while the overhead remains low. Increasing the number of allowed copies will not endlessly improve these statistics: the overhead grows rapidly in the binary case and there are not enough encounters for the nonbinary case to keep delivering better results.

The PRoPHET algorithms use historical information to route messages. In simulations that can be found in literature, there often is a warm-up period for history based protocols to build up an initial understanding of the network. In our scenario this is not possible: the simulation is in fact a whole festival day where visitors enter the terrain at a certain moment and actually have no knowledge of the other nodes or their history. Though the delivery ratios are relatively good, these protocols produce a large amount of overhead as can be seen in the figures. While the first version of the PRoPHET protocol performs better in terms of delivery as the $\beta$-parameter is increased, the second version responds inversely. Because the results of PRoPHETv2 with $\beta=0.09,0.25,0.90$ performed almost identically these results were omitted from the figures.

\section{Comparison}

When performing simulations with less than 500 nodes it becomes clear that the SW protocols are related to EP. Indeed, when enough copies of a message are allowed in SW (and especially in the binary case), messages can spread to almost all nodes like in EP. When fewer nodes are used in the simulation of the festival scenario, the crowd gets less connected and the spreading of the messages will be slowed down. This results in a higher latency for EP that is comparable to SW in the same setting, the latter with the advantage of requiring a limited amount of copies.

By comparing the findings of Keränen and Ott [1] to our results, it is again clear that the underlying mobility models play a very important role. While the goal of sending small messages in a DTN environment is the same, they state that disabling the transitivity property $(\beta=0.0)$ in the PRoPHET protocol yields the best results in a map-based and working day movement model, whereas the music festival mobility yields better results (in terms of delivery) when this property is actually used $(\beta=0.09,0.25,0.90)$.

Del Duca Almeida et al. [18] compared MANET and DTN routing protocols in three scenarios using the ns-2 simulator. They observe that the examined DTN protocols (Spray and Wait, Epidemic and PRoPHET) have almost the same end-toend latency, using other mobility patterns with a relatively low amount of nodes. After investigating the power requirements, they report the expected increase of energy usage as protocols send many copies of messages, as in Epidemic, or need a lot of control messages, like in PRoPHET. They only tested the binary Spray and Wait protocol with $n=6$ which has a much lower energy consumption while having a similar delivery ratio in comparison with the Epidemic or PRoPHET protocols. These findings confirm our assumptions regarding the energy requirements of the protocols.

\section{Candidate Selection}

To narrow down the candidate set, two representative scenarios are studied that stress different metrics of the protocols in the results of the simulations. The first is an emergency scenario, in which it is important that small messages reach their destination quickly and with a high degree of reliability. In these conditions, power consumption and overhead are nonessential factors to decide upon the 'best choice'. To clarify the performance of the protocols in this condition, figure 3(a) plots these variables in an X Y fashion. Protocols with desirable performance are ideally located in the lower righthand quadrant of the chart. Outliers (e.g. FC) are eliminated in these charts for reasons of clarity. In this condition EP is the obvious candidate as it clearly outperforms the others.

A second scenario describes a energy-conscious condition, in which the delivery of messages is less important, but in which users rather are focused on conserving their device's power source. This translates into the minimization of the overhead and optimization of the delivery ratio metrics. Figure 3(b) depicts precisely these variables; candidate protocols are found in the lower right-hand part of the chart.

Overall, based on our simulations using realistic node movement, we propose the Epidemic and binary Spray-andWait protocol as promising candidates for use at mass events. The main advantage of EP is the high delivery rate and low latency when the crowd is sufficiently interconnected, although the overhead is relatively high. The $\mathrm{SWb}$ protocols show similarities to the EP protocols yet limit the amount of copies in the network, thus lowering the overhead, while performing similar to EP in smaller networks. Both protocols can be tuned according to the specific context of use (e.g. the number of messages in the $\mathrm{SWb}$ case). Given the fact that the routing protocol and its parameters are implemented entirely in software, such a switch can easily be performed. Indeed, Spyropoulos et al. [15] describe that the Spray and Wait protocol can be tuned online to achieve the desired QoS requirements, providing a practical way for adapting the protocol to the current situation. The EP protocol could analogously be tuned by e.g. adjusting the sending frequency depending on the number of received messages. Furthermore, given the underlying mobility, the latency of the delivered messages is lower as in more advanced protocols like PRoPHET while similar or better delivery ratios are realized. Lastly, the amount of control messages needed for these protocols is relatively low.

\section{CONCLUSION AND FUtURE WORK}

In this paper we have compared simulations of opportunistic routing protocols in the context of a mass event, i.e. a music festival. The specific movement patterns of people at such an event were captured by placing Bluetooth detectors in 


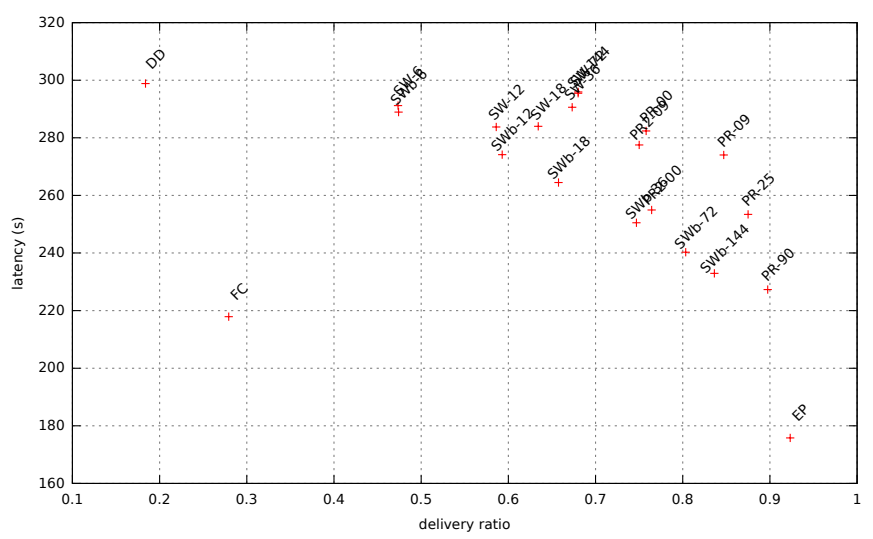

(a) Emergency condition

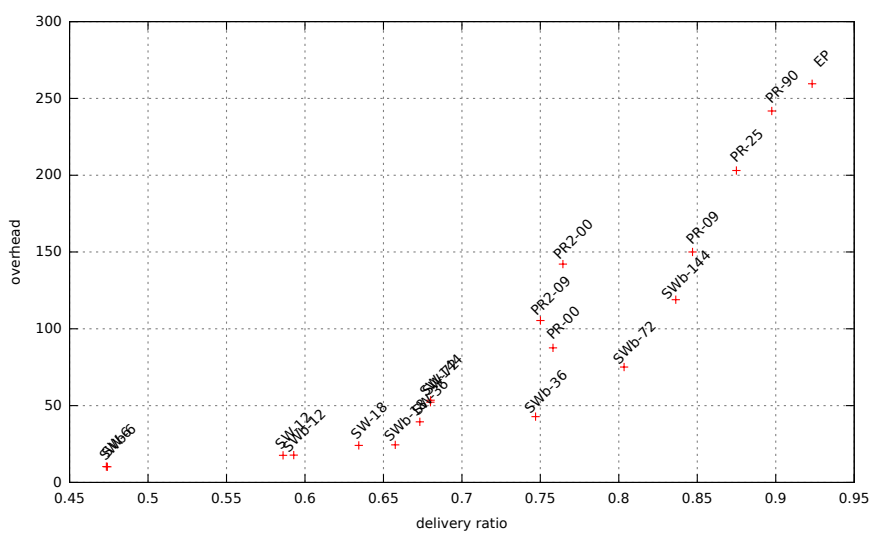

(b) Energy-conscious condition

Fig. 3. Scenario-based performance evaluation.

the festival area. The resulting data was transformed and used in the simulations to obtain realistic movement models. We have used four metrics to analyse and describe properties of the routing protocols. By highlighting and analyzing several important properties of the protocols, we have been able to compare the protocols. Based on the results, the Epidemic and binary Spray-and-Wait protocols are proposed as most suitable candidates as they perform similar to more advanced protocols in terms of delivery ratio, while keeping the overhead and need for control messages relatively low. Moreover, it is possible to tune parameters at run time to perform better in certain situations.

We plan to use the mobility traces for evaluating additional protocols and to perform simulations in other network simulators, like ns-3 or OMNeT++, in order to obtain more insights in the existing routing protocols. By using other simulators the results of the simulations in this paper can be confirmed and other metrics, e.g. energy requirements, can be studied.

The ultimate goal is to create and evaluate real applications in a similar scenario as described in this paper using the acquired insights from the simulations. The first envisioned application was already briefly described in this paper and will be designed to send and receive small messages at a mass event like a music festival. In this application a similar protocol as evaluated in the simulations will be used to route data packets in the network.

\section{ACKNOWLEDGMENT}

We would like to thank the Pukkelpop organization for allowing us to perform experiments during the three days of their festival.

\section{REFERENCES}

[1] A. Keränen and J. Ott, "Increasing Reality for DTN Protocol Simulations," Helsinki University of Technology, Tech. Rep., 2007.

[2] Z. Zhang, "Routing in intermittently connected mobile ad hoc networks and delay tolerant networks: overview and challenges," Communications Surveys Tutorials, IEEE, vol. 8, no. 1, pp. 24-37, 2006.

[3] M. Khabbaz, C. Assi, and W. Fawaz, "Disruption-tolerant networking: A comprehensive survey on recent developments and persisting challenges," Communications Surveys Tutorials, IEEE, vol. 14, no. 2, pp. 607-640, 2012.
[4] T. R. Henderson, S. Roy, S. Floyd et al., "ns-3 project goals," in Proc. ACM Workshop on ns-2: the IP network simulator (WNS2 '06), New York, NY, USA, 2006.

[5] A. Varga and R. Hornig, "An overview of the OMNeT++ simulation environment," in Proc. Int. Conf. on Simulation tools and techniques for communications, networks and systems \& workshops (SIMUTools '08), 2008.

[6] T. R. Andel and A. Yasinsac, "On the credibility of MANET simulations," Computer, vol. 39, no. 7, pp. 48-54, 2006.

[7] S. Grasic and A. Lindgren, "An analysis of evaluation practices for dtn routing protocols," in Proc. ACM Int. Workshop on Challenged Networks (CHANTS '12), New York, NY, USA, 2012, pp. 57-64.

[8] N. Aschenbruck, A. Munjal, and T. Camp, "Trace-based mobility modeling for multi-hop wireless networks," Computer Communications, vol. 34, no. 6, pp. 704-714, May 2011.

[9] J. Yeo, D. Kotz, and T. Henderson, "CRAWDAD: A Community Resource for Archiving Wireless Data at Dartmouth," ACM SIGCOMM Computer Communication Review, vol. 36, no. 2, pp. 21-22, Apr. 2006.

[10] K. Lee, S. Hong, S. J. Kim et al., "SLAW: A New Mobility Model for Human Walks," in IEEE INFOCOM '09, 2009, pp. 855-863.

[11] M. Radenkovic and A. Grundy, "Framework for utility driven congestion control in delay tolerant opportunistic networks," in Proc. IEEE Wireless Communications and Mobile Computing Conference (IWCMC '11), july 2011, pp. $448-454$.

[12] M. Versichele, T. Neutens, M. Delafontaine et al., "The use of Bluetooth for analysing spatiotemporal dynamics of human movement at mass events: A case study of the Ghent Festivities," Applied Geography, vol. 32, no. 2, pp. 208-220, 2012.

[13] V. Vukadinovic and S. Mangold, "Opportunistic wireless communication in theme parks: a study of visitors mobility," in Proc. ACM Int. Workshop on Challenged Networks (CHANTS'11), New York, NY, USA, 2011, pp. 3-8.

[14] A. Vahdat and D. Becker, "Epidemic Routing for Partially Connected Ad Hoc Networks," Duke University, Tech. Rep., 2000.

[15] T. Spyropoulos, K. Psounis, and C. S. Raghavendra, "Spray and wait: an efficient routing scheme for intermittently connected mobile networks," in Proc. ACM SIGCOMM Workshop on Delay-tolerant networking (WDTN'05), New York, NY, USA, 2005, pp. 252-259.

[16] A. Lindgren, A. Doria, and O. Schelén, "Probabilistic routing in intermittently connected networks," ACM SIGMOBILE Mobile Computing and Communications Review, vol. 7, no. 3, p. 19, Jul. 2003.

[17] S. Grasic, E. Davies, A. Lindgren et al., "The evolution of a DTN routing protocol - PRoPHETv2," in Proc. ACM Int. Workshop on Challenged Networks (CHANTS'11), New York, NY, USA, 2011, pp. 27-30.

[18] V. Del Duca Almeida, A. B. Oliveira, D. F. Macedo et al., "Performance evaluation of MANET and DTN routing protocols," in Wireless Days (WD), 2012 IFIP, Dublin, Ireland, Nov. 2012, pp. 1-6. 